\title{
HOMOMORPHISMS AND MODULAR FUNCTIONALS
}

\author{
BY \\ SAUL GORN
}

This paper is concerned with complemented modular lattices containing the elements $O$ and $I$. The first part treats of homomorphisms of the lattice $L$, their existence, determination and invariant properties. The second considers norms (i.e., sharply positive or, alternatively, strictly monotone modular functionals) and quasi-norms (i.e., positive or monotone modular functionals) on $L$, their interconnections, and necessary and sufficient conditions for their unicity up to linear transformations.

There are six main parts to the paper, as follows:

1. The homomorphism theorem. The dual concepts of $\sigma$-ideal and $\pi$-ideal are defined for general lattices. Duality is essential throughout the paper. The $C$-operator, which takes all elements of a subset of $L$ into their complements, is introduced, and $C$-neutral ideals are defined as those which appear in complementary pairs $a, \bar{a}$. Theorems 1 and 2 state that any $C$-neutral pair of ideals determine a congruence in $L$ by means of any one of six equivalent conditions. These conditions are recognizable as those appearing in Boolean algebra, but the proof of their equivalence in the general case considered here is far from trivial, since it requires the fundamental Lemma 7 . Theorem 3 states that all congruences are thus obtained from $C$-neutral ideals. Quotient lattices $L / \mathfrak{a}$ are defined, and it is obvious that every homomorph of $L$ is equivalent to an $L / \mathfrak{a}$. For example, consider a regular Carathéodory measure in a metric space, the measure of the space being 1. In the Boolean algebra of measurable sets, the sets of measure 0 and measure 1 are complementary $C$-neutral ideals, the first $\sigma$, the second $\pi$, and the quotient lattice is isomorphic with a sublattice of the $G_{\delta}$ 's.

2. The preservation of normal ideals under homomorphism. The operators $c_{\sigma}, c_{x}$, and ', are defined. By means of the first two we define normal ideals, the upper and lower segments of MacNeille's cuts, whose main reason for existence is to make up for the "gaps" when $L$ is not complete. The main theorem (Theorem 7) states that a homomorphism preserves normality for $\mathfrak{b} \supseteq \mathfrak{a}$; and the pre-image of a normal ideal is normal if $\mathfrak{a}$ is normal. The preliminaries to Theorem 7 state in effect that the operators $C, c$, and ' preserve complementary $C$-neutrality for pairs of ideals, yielding by iteration at most three pairs from a given $\mathfrak{a}$ and $\overline{\mathfrak{a}}$. It follows that normality in our definition is a proper generalization of Stone's in a Boolean algebra. Lemma 12 gives a connection between neutrality and distributivity parallel to that for complementary neutral elements, $a, \bar{a}$.

Presented to the Society, October 26, 1940; received by the editors December 5, 1940. 
3. Ideal reducibility. This concept generalizes that of reducibility. Essentially it means that if $L$ were complete, it would be reducible. $L / \mathfrak{a}$ is ideally irreducible if and only if $\mathfrak{a}$ is maximal (Theorem 8-prime, for $\pi$-ideals).

4. Relationship between norms and quasi-norms. Every quasi-norm determines a complementary pair of $C$-neutral ideals, $\mathfrak{a}, \overline{\mathfrak{a}}$, the elements whose quasi-norms are 0 and 1 (Theorem 9); furthermore (Theorem 10) the quasinorm determines a norm in $L / \mathfrak{a}$, and every quasi-norm is determined by the $\mathfrak{a}$ and the norm in $L / \mathfrak{a}$.

5. Unicity of norm. If $L$ is reducible, its norm is not unique (Lemma 15). This result is elementary and is probably well known. The converse is false; ideal irreducibility is needed. As an example of 4 and 5 , since the only irreducible Boolean algebra is $\{O, I\}$, the measure function in 1 is unique if and only if every measurable set is either of measure 0 or of measure 1 . The WilcoxSmiley continuity conditions on a norm are introduced. Essentially, they say that none of the gaps in $L$ affects the norm. If a norm satisfies these conditions, then (Theorems 11 and 12) $L$ is ideally irreducible if and only if the norm is unique, which in turn is true if and only if the complete envelope of $L$ is irreducible. von Neumann's theorem on the unicity of dimension in a continuous geometry is needed in the proof.

6. Normality. If a quasi-norm in $L$ satisfies the W.S. conditions, then (Theorem 13) the ideals determined as in 4 are normal. Hence (Theorem 14) the quasi-norm is unique, by 3 , if and only if $a$ is maximal or prime.

By a $\pi$-ideal $a$ in $L$, we mean a set of elements such that $a, b \in \mathfrak{a}$ imply $a b \in \mathfrak{a}$, and such that $a \in \mathfrak{a}, c \geqq a$ imply $c \in \mathfrak{a}$. The dual of this statement defines a $\sigma$-ideal. The letters $\mathfrak{a}$ and $\mathfrak{b}$ refer exclusively to ideals.

If $A \subseteq L$, then by $C A$ we mean the set of all complements of all the individual $a \in A$. If $A=\{a\}$, we write $C a$ instead of $C A$. Note that $C C A \supseteq A$. By means of Lemma 2 below, it is easy to see that if $\mathfrak{a}$ is a $\pi$-ideal, $C \mathfrak{a}$ is the set of all $x$ for which $x a=O$ for some $a \in \mathfrak{a}$; the dual statement holds for $\sigma$-ideals.

We call $\mathfrak{a}$ a $C$-ideal if $C \mathfrak{a}$ is a $\pi$-or $\sigma$-ideal. Note that if $\mathfrak{a}$ is a $C$-ideal and a $\pi$-ideal, then $C \mathfrak{a}$ is a $\sigma$-ideal, and vice versa.

We shall never use $a^{\prime}$ to indicate a complement of an element. On the other hand, a bar over a letter will always indicate that we have before us a uniquely determined complement.

An ideal $\mathfrak{a}$ is called neutral (the terminology is due to G. Birkhoff, p. 59, footnote) if $a^{\prime} \in \mathfrak{a}$ whenever $a \in \mathfrak{a}$ and $a^{\prime}$ has a common complement with $a$ (i.e., whenever $a$ and $a^{\prime}$ are perspective, and hence projective); in other words, $\mathfrak{a}$ is neutral if $C C \mathfrak{a} \subseteq \mathfrak{a}$. Thus, $\mathfrak{a}$ is neutral if and only if it is $C C$-closed, i.e., $C C \mathfrak{a}=\mathfrak{a}$. Notice that in this definition $C \mathfrak{a}$ need not be an ideal. We leave the relationship between neutral and $C$-ideals an open problem and define a $C$-neutral ideal as a neutral ideal which is also a $C$-ideal.

It is easy to see that for principal ideals, $\mathfrak{a}(a)$ is neutral if and only if $a$ is neutral, which is the case if and only if $\mathfrak{a}(a)$ is a $C$-ideal. For principal ideals 
the $C$-ideals and the neutral ideals are the same. Only Lemmas 3 and 6 below are needed to prove these statements.

If $\mathfrak{a}$ is $C$-neutral, we write $\bar{a}$ instead of $C \mathfrak{a}$. If $a$ is neutral, we write $\bar{a}$ for its unique complement. By the complement of $a$ in $b$, where $a<b$, we mean the complement of $a$ in $L(O, b)$; dually, by a complement of $a$ over $b$, where $a>b$, we mean a complement of $a$ in $L(b, I)$.

LEMMA 1. If $a \geqq b \geqq c \geqq d$ and $r$ is a complement of $b$ in $L(a, c)$ and $s$ is $a$ complement of $c$ in $L(a, d)$, then rs is a complement of $b$ in $L(a, d)$. Dually, we get a corresponding theorem using $a \leqq b \leqq c \leqq d, r+s$, and $L(c, a), L(d, a)$.

For, by hypothesis, we have $a \geqq r \geqq c, a \geqq s \geqq d, b+r=a, b r=c$, and $s+c=a$, $s c=d$; hence,

and

$$
d=s c=s(r b)=(r s) b,
$$

$$
\begin{aligned}
b+r s & =r s+b(s+c)=r s+b s+c=(r s+c)+(b s+c) \\
& =r(s+c)+b(s+c)=r+b=a .
\end{aligned}
$$

LEMma 2. If $x a=O$, then there is $a b \in C a$ with $b \geqq x$.

For, let $z \in C(x+a)$; then the dual of Lemma 1 with $O \leqq a \leqq x+a \leqq I, x$ for $r$, and $z$ for $s$, gives us $x+z \in C a$.

LEMmA 3. If $a, a^{\prime} \in C b$ and $a^{\prime} \leqq a$, then $a=a^{\prime}$.

For, since $I=a^{\prime}+b$ and $O=a b, a=a\left(a^{\prime}+b\right)=a^{\prime}+a b=a^{\prime}$.

Lemma 4. If $x \leqq a$ and $b \in C a$, then there is $a y \in C x$ for which $y \geqq b$.

For, since $a b=O, x b \leqq a b=O$. It therefore follows, by Lemma 2 , that there is a $y \in C x$ with $y \geqq b$.

LEMmA 5. If $a_{1} \leqq a_{2} \leqq a_{3}$ and $b_{1} \geqq b_{3}$ with $b_{i} \in C a_{i}$, then there is a $b_{2} \in C a_{2}$ with $b_{1} \geqq b_{2} \geqq b_{3}$. Then

For, by Lemma 4 , we may take $c_{2} \leqq b_{1}$ with $c_{2} \in C a_{2}$ and $b_{2}=b_{3}+a_{3} c_{2} \leqq b_{1}$.

and

$$
a_{2}+b_{2}=a_{2}+a_{3} c_{2}+b_{3}=a_{3}\left(a_{2}+c_{2}\right)+b_{3}=a_{3}+b_{3}=I,
$$

$$
a_{2} b_{2} \leqq a_{3} b_{2}=a_{3}\left(b_{3}+a_{3} c_{2}\right)=a_{3} b_{3}+a_{3} c_{2}=a_{3} c_{2} ;
$$

so that $a_{2} b_{2} \leqq a_{3} c_{2} a_{2}=O$. Consequently $a_{2} b_{2}=O$.

Lemma 6. If $b \leqq a$ and $a$ is neutral, then $c \geqq \bar{a}$ for any $c \in C b$. Dually, if $b \geqq a$ and $a$ is neutral, then $c \leqq \bar{a}$ for any $c \in C b$.

For $\bar{a} b \leqq \bar{a} a=O$ and $I=b+c$. Hence $\bar{a}=\bar{a} b+\bar{a} c=\bar{a} c$. In the dual, note that for neutral elements $\bar{a}$ we have $\bar{a}=(\bar{a}+b)(\bar{a}+c)$. 
Lemma 7. If $\mathfrak{a}$ is a $C$-neutral $\pi$-ideal, then for any $x \in L$ and $t \in \mathfrak{a}$, any complement of $t x$ in $x$ is in $\vec{a}$. Dually, if $\mathfrak{a}$ is a C-neutral $\sigma$-ideal, then, for any $x \in L$ and $t \in \mathfrak{a}$, any complement of $t+x$ over $x$ is in $\overline{\mathfrak{a}}$.

For let $u$ be a complement of $t x$ in $x$ and $y \in C x$; then an application of Lemma 1 to $O \leqq t x \leqq x \leqq I$ gives $u+y \in C(t x)$. It follows that $O=t x(u+y)$ $=t(u+x y)=t u$. Hence $u \in \overline{\mathfrak{a}}$.

TheOREM 1. If a is a $C$-neutral $\pi$-ideal and $\overline{\mathfrak{a}}=C \mathfrak{a}$, then the following relations between $a$ and $b$ are equivalent:

1. There is $a t \in \mathfrak{a}$ with $a b=(a+b) t$.

$1^{\prime}$. There is a $u \in \overline{\mathfrak{a}}$ with $a+b=a b+u$.

2. There is a $t \in \mathfrak{a}$ with $a t=b t$.

$2^{\prime}$. There is $a u \in \overline{\mathfrak{a}}$ with $a+u=b+u$.

3. There is a $t \in \mathfrak{a}$ and $a u \in \overline{\mathfrak{a}}$ with $a=b t+u$.

$3^{\prime}$. There is a $t \in \overline{\mathfrak{a}}$ and $a u \in \mathfrak{a}$ with $a=(b+t) u$.

By duality, we need only consider the proofs that $1^{\prime}$ follows from 1 , and that 1 implies 2, which in turn implies 3 , which finally implies 1 .

In proving that $1^{\prime}$ follows from 1 , we may assume that there is a $t \in a$ for which $a b=(a+b) t$, and must find a $u \in \overline{\mathfrak{a}}$ for which $a+b=a b+u$. Using $a+b$ for $x$ in Lemma 7 , let $u$ be a complement of $(a+b) t$ in $a+b ; u \in \bar{a}$. Hence $a b+u=a+b$.

Condition 2 follows from 1: $a t=a(a+b) t=a a b=a b=b a b=b(a+b) t=b t$. To show that 2 implies 3 , let $u$ be a complement of $a t$ in $a ; a=b t+u$. But, by Lemma $7 u \in \overline{\mathfrak{a}}$.

The proof that 3 implies 1 presents the greatest difficulty. We are given a $t \in \mathfrak{a}$ for which $a=b t+u$, where $u \in \overline{\mathfrak{a}}$, and must find an element of $\mathfrak{a}$ which gives $a b$ when multiplied by $a+b$. Since $a=b t+u, a+b=b+u$. Applying Lemma 7 to $b+u$ over $b$ yields a $t_{1} \in \mathfrak{a}$ with $(b+u) t_{1}=b$, i.e., $(a+b) t_{1}=b$. Now apply Lemma 7 to $a=b t+u$ over $b t$; we get a $t_{2} \in \mathfrak{a}$ for which $a t_{2}=(b t+u) t_{2}=b t$. Another application of Lemma 7, this time to $b t$ under $b$, gives us a $u_{2} \in \bar{a}$ with $a t_{2}+u_{2}=b t+u_{2}=b$. It follows that $a+b=a+u_{2}$. This permits us a final application of Lemma 7 , to $a+u_{2}$ over $a$, providing a $t_{3} \in \mathfrak{a}$ for which $(a+b) t_{3}=\left(a+u_{2}\right) t_{3}=a$. Using this last equation with the corresponding expression for $b$ (from our first use of Lemma 7), we get $(a+b) t_{1} t_{3}=(a+b) t_{1}(a+b) t_{3}$ $=b a$, where $t_{1} t_{3} \in \mathfrak{a}$ since $t_{1}, t_{3} \in \mathfrak{a}$.

THEOREM 2. Define $a \equiv b$ to mean that any one of the six equivalent conditions of Theorem 1 holds between $a$ and $b$. Then $\equiv$ is a congruence relation, the elements $\equiv I$ form a, and the elements $\equiv O$ form $\overline{\mathfrak{a}}$.

For, since $a I=a I, a \equiv a$; if $a t=b t$, then $b t=a t$, so that $a \equiv b$ implies $b \equiv a$. If $a \equiv b \equiv c$, then $a t_{1}=b t_{1}$ and $b t_{2}=c t_{2}$, where $t_{i} \in \mathfrak{a}$; it follows that $t_{1} t_{2} \in \mathfrak{a}$ and $a t_{1} t_{2}=c t_{1} t_{2}$. Hence $a \equiv c$. 
Suppose $a \equiv b$ and $c \equiv d$. On the one hand $a t_{1}=b t_{1}, c t_{2}=d t_{2}$, where $t_{i} \in a$; $t_{1} t_{2} \in \mathfrak{a}$ and $a c t_{1} t_{2}=b d t_{1} t_{2}$, hence $a c \equiv b d$. On the other hand, $a+u_{1}=b+u_{1}$, $c+u_{2}=d+u_{2}$, where $u_{i} \in \overline{\mathfrak{a}} ; u_{1}+u_{2} \in \overline{\mathfrak{a}}$ and $(a+c)+\left(u_{1}+u_{2}\right)=(b+d)+\left(u_{1}+u_{2}\right)$, hence $a+c \equiv b+d$.

Finally, $a \equiv I, a t=I t$ for some $t \in \mathfrak{a}, a t=t$ for some $t \in \mathfrak{a}, a \geqq t$ for some $t \in \mathfrak{a}$, and $a \in \mathfrak{a}$ are equivalent conditions.

THEOREM 3. If $\theta$ is a congruence in $L$, then the set of elements $\equiv I$ is a $C$-neutral $\pi$-ideal $\mathfrak{a}$, and the set of elements $\equiv O$ is the $C$-neutral $\sigma$-ideal $\overline{\mathfrak{a}}$, and $\theta$ is the same as the congruence determined by $\mathfrak{a}($ or $\overline{\mathfrak{a}})$ in Theorem 2. (This is an amplification of Birkhoff's Theorem 4.3.)

If $a \equiv I$ and $b \geqq a$, then $b \equiv I$; if $a \equiv I$ and $b \equiv I$, then $a b \equiv I$; hence the elements $\equiv I$ form a $\pi$-ideal, $a$; the dual statement holds for the elements $\equiv O$. But if $a \equiv I$ and $a b=O$, then $O=a b \equiv I b=b$; hence the elements $\equiv O$ form the set $C a$. which is therefore an ideal, and $a$ is a $C$-ideal; but dually we have $\mathfrak{a}=C C \mathfrak{a}$, so that $\mathfrak{a}$ is neutral. Now if $a \equiv b(\theta)$, then $a b \equiv a+b(\theta)$; take $t$ a complement of $a b$ in $a+b$, so that $a b+t=a+b$, and $a b t=O$; condition $1^{\prime}$ of Theorem 1 then follows, since $t=(a+b) t \equiv a b t=O$. Hence $a \equiv b(\mathfrak{a})$. If $a \equiv b(\mathfrak{a})$, then there is a $u \in \mathfrak{a}$ for which $a+u=b+u ; u \equiv O(\theta)$, hence $a \equiv a+u=b+u \equiv b(\theta)$.

Corollary. If $L$ is a complemented modular lattice, then the congruences in $L$ are in $(1,1)$ correspondence with the $C$-neutral $\pi$-ideals $(\sigma$-ideals) in $L$.

The proof is obvious.

If $\mathfrak{a}$ is $C$-neutral, we define $A_{a}$ to mean the set of all $x \equiv a(\mathfrak{a})$; we also define $L / \mathfrak{a}$ to mean the set of all $A_{a}$, where $A_{a}+A_{b}=A_{a+b}, A_{a} A_{b}=A_{a b}$, and hence $A_{a} \leqq A_{b}$ if and only if $a b \equiv a(\mathfrak{a})$. Note that $L / \mathfrak{a}$ is a complemented, modular lattice with $A_{o}$ and $A_{I}$ for $O$ and $I$. $A^{*}$ is defined to mean the set of all $A_{a}$ for which $a \in A$.

CoROllaRY. If $L$ is a complemented, modular lattice and is homomorphic to the lattice $L^{\prime}$, then $L^{\prime}$ has an $O$ and $I$, the elements of $L$ homomorphic to $O$ form a C-neutral $\sigma$-ideal a, those homomorphic to $I$ form $\overline{\mathfrak{a}}$, and $L^{\prime}$ is isomorphic to $L / \mathfrak{a}$.

The proof is obvious, since " $a$ and $b$ have the same homomorph" is a congruence relation.

Lemma 8. If $d^{*} \in C a^{*}$, then there is a $b \in C a$ for which $b^{*}=d^{*}$. The converse is obvious.

For if the congruence ideals are $\mathfrak{a}, \overline{\mathfrak{a}}$, the first being a $\sigma$-ideal, then $(a d)^{*}=a^{*} d^{*}=O^{*}$, i.e., $a d \in a$. Take $t \in C a d$, so that $t \in \bar{a}$; then $d t \equiv d$, and $a(d t)=(a d) t=O$. By Lemma 2 there is a $b \geqq d t$ with $b \in C a$; therefore $b=b+d t \equiv b+d$ and consequently $b^{*}=(b+d)^{*}=b^{*}+d^{*}$. Thus $b^{*} \geqq d^{*}$ and $b^{*}, d^{*} \in C a^{*}$. We may now apply Lemma 3 in $L^{*}=L / \mathfrak{a}$ to give $b^{*}=d^{*}$. 
THEOREM 4. There is a $(1,1)$ correspondence between the ideals of $L, \mathfrak{b} \supseteq \mathfrak{a}$, and the ideals of $L^{*}, \mathcal{B}$, given by

$$
\mathbb{B}=\mathfrak{b}^{*}, \quad \mathfrak{b}=\mathfrak{b}^{*} \in \mathcal{B}^{b^{*}}
$$

Thus, if $\mathfrak{b}_{1}^{*}=\mathfrak{b}_{2}^{*}$ and $\mathfrak{b}_{i} \supseteq \mathfrak{a}$, then $\mathfrak{b}_{1}=\mathfrak{b}_{2}$. This correspondence is also $(1,1)$ for the C-neutral ideals.

In our proof we may restrict ourselves to $\pi$-ideals. Let the ideal $\mathfrak{b} \supseteq \mathfrak{a}$, $b \in \mathfrak{b}$, and $d \equiv b$; then $d t=b t$ for some $t \in \mathfrak{a} \subseteq \mathfrak{b}$. Hence $d \geqq d t=b t \in \mathfrak{b}$, so that $d \in \mathfrak{b}$. Thus any ideal $\supseteq \mathfrak{a}$ contains complete congruence classes, and (1) follows. If $a^{*} \geqq b^{*}$ and $b^{*} \in b^{*}$, then $(a b)^{*}=a^{*} b^{*}=b^{*}$, and $a b \equiv b$; there is consequently a $t \in \mathfrak{a}$ with $a b t=b t$. It therefore follows that $a \geqq b t \in \mathfrak{b}, a \in \mathfrak{b}$, and $a^{*} \in \mathfrak{b}^{*}$. If $a^{*}, b^{*} \in \mathfrak{b}^{*}$, then $a b \in \mathfrak{b}$, and $a^{*} b^{*}=(a b)^{*} \in \mathfrak{b}^{*}$. Thus $\mathfrak{b}^{*}$ is an ideal. If $\mathcal{B}$ is an ideal in $L^{*}$ and (1) holds, then $\mathfrak{b}$ is an ideal $\supseteq \mathfrak{a}=I^{*}$, since $I^{*} \in \mathcal{B}$, and the typical argument holds, namely: if $b_{1}, b_{2} \in \mathfrak{b}$, then $b_{i}^{*} \in \mathcal{B}$ so that $\left(b_{1} b_{2}\right)^{*} \in \mathcal{B}$, and hence $b_{1} b_{2} \in \mathfrak{b}$; if $b_{1} \geqq b_{2} \in \mathfrak{b}$, then $b_{1}{ }^{*} \geqq b_{2}{ }^{*} \in \mathcal{B}, b_{1}^{*} \in \mathcal{B}$, and consequently $b_{1} \in \mathfrak{b}$.

Suppose $\mathfrak{b}$ is $C$-neutral. Then $C \mathfrak{b}^{*}=$ the set of $C b^{*}=$ the image of the $C b$ (by Lemma 8$)=(C \mathfrak{b})^{*}$; since $C \mathfrak{b}$ is a $\sigma$-ideal, so is $C \mathfrak{b}^{*}$ by the dual of the preceding; it follows that $\mathfrak{b}^{*}$ is a $C$-ideal.

The dual argument yields $C C \mathfrak{b}^{*}=C(C \mathfrak{b})^{*}=(C C \mathfrak{b})^{*}=\mathfrak{b}^{*} ; \mathfrak{b}^{*}$ is $C$-neutral.

The same reasoning gives: if $\mathfrak{b}^{*}$ is $C$-neutral, $(C \mathfrak{b})^{*}$ is an ideal, and $\mathfrak{b}^{*}=C C \mathfrak{b}^{*}=(C C \mathfrak{b})^{*}$; hence to prove $\mathfrak{b} C$-neutral, we have only to show that the pre-images of $(C \mathfrak{b})^{*}$ and $(C C \mathfrak{b})^{*}$ are $C \mathfrak{b}$ and $C C \mathfrak{b}$; i.e., that if $x \in C \mathfrak{b}$ and $y \equiv x$, then $y \in C \mathfrak{b}$, and if $x \in C C \mathfrak{b}$ and $y \equiv x$, then $y \in C C \mathfrak{b}$. If $x \in C \mathfrak{b}$ and $y \equiv x$, then $y^{*}=x^{*} \in C \mathfrak{b}^{*}$. Lemma 8 then gives us an $a \in C y$ for which $a \equiv b$. Thus $y \in C a$ and $a \in \mathfrak{b}$. Hence $y \in C \mathfrak{b}$ and $C \mathfrak{b}$ is an ideal. The dual argument makes $C C \mathfrak{b}$ an ideal. Now $C C \mathfrak{b} \supseteq \mathfrak{a}$, so that, since $\mathfrak{b}^{*}=(C C \mathfrak{b})^{*}, C C \mathfrak{b}=\mathfrak{b}$.

If $A \subseteq L$, we define $c_{\sigma} A$ to mean the set of all $x \in L \leqq$ every $a \in A$, and $c_{\pi} A$ to mean the set of all $x \in L \geqq$ every $a \in A$. A $\sigma$-ideal ( $\pi$-ideal) is normal $\left({ }^{1}\right)$ if $\mathfrak{a}=c_{\sigma} c_{\pi} \mathfrak{a}\left(\mathfrak{a}=c_{\pi} c_{\sigma} \mathfrak{a}\right)$.

The $c_{\sigma} A, c_{\pi} A$ are normal. The normal ideals of either type form the complete envelope of $L$, being the lower and upper segments of MacNeille's cuts.

LEMMA 9. $a=$ g.l.b. $a^{*}$ if and only if $a \in c_{\sigma} \bar{a} ; a=1$.u.b. $a^{*}$ if and only if $a \in c_{\pi} \mathfrak{a}$, where $a$ is $a C$-neutral $\sigma$-ideal. If $b \in C a$, then $a=\mathrm{g} .1 . \mathrm{b} . a^{*}$ if and only if $b=$ l.u.b. $b^{*}$.

The proof divides naturally into three parts:

1. If $a \in c_{\sigma} \overline{\mathfrak{a}}$ and $b \equiv a$, then there is a $t \in \overline{\mathfrak{a}}$ for which $a t=b t$; since $a \leqq t$, it follows that $a=b t$ and $a \leqq b$.

(1) For the introduction of these ideas see the author's abstract 45-1-17; Wilcox and Smiley's Metric lattices in Annals of Mathematics, (2), vol. 40 (1939), and \$33 in Birkhoff. 
2. If $a \equiv b$ implies $a \leqq b$, then for any $t \in \bar{a}$ we have, as a consequence of $a \equiv a t, a \leqq a t$; thus $a=a t$, and $a \leqq t$.

3. Suppose $a=$ g.l.b. $a^{*}$ and $b \in C a$, but $b \neq 1$.u.b. $b^{*}$, so that part 2 permits us to say that there is a $u \in \mathfrak{a}$ with $b+u>u$. By Lemmas 3 and 4 there is an $a_{1}<a$ with $b+u \in C a_{1}$. It follows that $a_{1} b=O$ and $a_{1}+b \equiv a_{1}+b+u=I$; hence Lemma 8 yields an $a_{2} \in C b$ for which $a_{2} \equiv a_{1}$, say $a_{2} t=a_{1} t$ with $t \in \bar{a}$. Then $a_{2} t \leqq a_{1}<a$. But

$$
a=a\left(b+a_{2}\right) \equiv a\left(b+a_{2} t\right)=a b+a_{2} t=a_{2} t .
$$

This contradicts the fact that $a=$ g.l.b. $a^{*}$.

The proof is then completed by appealing to duality.

Corollary. $C c_{\sigma}=c_{\pi} C, C c_{\pi}=c_{\sigma} C$. In other words, for any $C$-neutral $\sigma$-ideal a we have

$$
C c_{\sigma} \overline{\mathfrak{a}}=c_{\boldsymbol{x}} \mathfrak{a}, \quad C c_{\boldsymbol{\pi}} \mathfrak{a}=c_{\sigma} C \mathfrak{a}=c_{\sigma} \overline{\mathfrak{a}} .
$$

For Lemma 9 gives us: if $b \in C a$, then $a \in c_{\sigma} \bar{a}$ if and only if $b \in c_{\pi} \mathfrak{a}$. Hence $c_{\sigma} \overline{\mathfrak{a}}$ and $c_{\boldsymbol{\pi}} \mathfrak{a}$ are complementary $C$-ideals.

Theorem 5. If $\mathfrak{a}$ is a $C$-neutral $\sigma$-ideal, then $c_{\sigma} \overline{\mathfrak{a}}$ and $c_{\pi} \mathfrak{a}$ are complementary $C$-neutral ideals, and $c_{\pi} c_{\sigma} \bar{a}$ and $c_{\sigma} c_{x} \mathfrak{a}$ are complementary $C$-neutral normal ideals containing $\overline{\mathfrak{a}}$ and $\mathfrak{a}$, respectively.

For $c_{\sigma} \overline{\mathfrak{a}}, c_{\pi} \mathfrak{a}$ are $C$-ideals, and

$$
C C c_{\pi} \mathfrak{a}=C c_{\sigma} \overline{\mathfrak{a}}=c_{\pi} C \overline{\mathfrak{a}}=c_{\pi} \mathfrak{a} ;
$$

dually for $c_{\sigma} \overline{\mathfrak{a}} ;$ hence $c_{\boldsymbol{\pi}} \mathfrak{a}$ and $c_{\sigma} \overline{\mathfrak{a}}$ are complementary $C$-neutral ideals for any $C$-neutral $\mathfrak{a}, \overline{\mathfrak{a}}$. Applying this to $c_{\pi} \mathfrak{a}$ and $c_{\sigma} \overline{\mathfrak{a}}$ instead of $\overline{\mathfrak{a}}$ and $\mathfrak{a}$, we get the remaining parts of the theorem.

With Stone we define: if $A \subseteq L$, then by $A^{\prime}$ we mean the set of all $b \in L$ for which

Case 1. $a b=O$ for all $a \in A$ if $A$ is $a \sigma$-ideal.

Case 2. $a+b=I$ for all $a \in A$ if $A$ is a $\pi$-ideal.

Note that this operation is a polarity (see Birkhoff $\S 32$ ), so that, similarly to $c_{\sigma}$ and $c_{x}$, we have: If $A \subseteq B$, then

$$
A^{\prime} \supseteq B^{\prime}, \quad A \subseteq A^{\prime \prime}, \quad A^{\prime \prime \prime}=A^{\prime} .
$$

Theorem 6. If a is a C-neutral $\sigma$-ideal, then

$$
\mathfrak{a}^{\prime}=c_{\sigma} \overline{\mathfrak{a}}=\left(c_{\sigma} \overline{\mathfrak{a}}\right)^{\prime \prime}=\left(c_{\sigma} \boldsymbol{C}_{\boldsymbol{\pi}} \mathfrak{a}\right)^{\prime}
$$

and

$$
\mathfrak{a}^{\prime \prime}=\left(c_{\sigma} \overline{\mathfrak{a}}\right)^{\prime}=\left(c_{\sigma} c_{\pi} \mathfrak{a}\right)^{\prime \prime}=c_{\sigma} c_{\pi} \mathfrak{a} .
$$

We will prove part of the theorem directly and will obtain the rest by continued application of the polarity properties. 
1. If $v \in c_{\sigma} \overline{\mathfrak{a}}$ and $u \in c_{\sigma} c_{\pi} \mathfrak{a}$, take $w \in C v$. Then $w \in c_{\pi} \mathfrak{a}$, so that $u \leqq w ;$ therefore $u v \leqq w v=O$, and $v \in\left(c_{\sigma} c_{\pi} \mathfrak{a}\right)^{\prime}, u \in\left(c_{\sigma} \overline{\mathfrak{a}}\right)^{\prime}$; i.e.,

$$
c_{\sigma} \overline{\mathfrak{a}} \subseteq\left(c_{\sigma} c_{\boldsymbol{\pi}} \mathfrak{a}\right)^{\prime}, \quad c_{\sigma} c_{\boldsymbol{\pi}} \mathfrak{a} \subseteq\left(c_{\sigma} \overline{\mathfrak{a}}\right)^{\prime} .
$$

2. If $v \in \mathfrak{a}$, let $t \in \bar{a}$ and let $u$ be a complement of $v t$ in $v$; by Lemma 7 we have $u \in \mathfrak{a}$ and $v=v t+u$. Now $u v=O$, therefore $u=u v=O, v=v t$, and hence $v \leqq t$. It therefore follows that $v \in c_{\sigma} \overline{\mathfrak{a}}$ and $\mathfrak{a}^{\prime} \subseteq c_{\sigma} \overline{\mathfrak{a}}$.

3. We now have $\mathfrak{a}^{\prime} \subseteq c_{\sigma} \overline{\mathfrak{a}} \subseteq\left(c_{\sigma} c_{\pi} \mathfrak{a}\right)^{\prime}$ and $c_{\sigma} c_{\pi} \mathfrak{a} \subseteq\left(c_{\sigma} \overline{\mathfrak{a}}\right)^{\prime}$; the last yields $\left(c_{\sigma} \overline{\mathfrak{a}}\right)^{\prime \prime} \subseteq\left(c_{\sigma} c_{\pi} \mathfrak{a}\right)^{\prime}$, hence

$$
\mathfrak{a}^{\prime} \subseteq c_{\sigma} \overline{\mathfrak{a}} \subseteq\left(c_{\sigma} \overline{\mathfrak{a}}\right)^{\prime \prime} \subseteq\left(c_{\sigma} c_{\boldsymbol{\pi}} \mathfrak{a}\right)^{\prime} ;
$$

therefore

$$
\mathfrak{a}^{\prime \prime} \supseteq\left(c_{\sigma} \overline{\mathfrak{a}}\right)^{\prime} \supseteq\left(c_{\sigma} c_{\pi} \mathfrak{a}\right)^{\prime \prime} \supseteq c_{\sigma} c_{\boldsymbol{\pi}} \mathfrak{a} .
$$

Now use $c_{\sigma} \overline{\mathfrak{a}}$ in the preceding instead of $\mathfrak{a}$ :

$$
\begin{gathered}
\left(c_{\sigma} \overline{\mathfrak{a}}\right)^{\prime} \subseteq c_{\sigma} c_{\boldsymbol{\pi}} \mathfrak{a} \subseteq\left(c_{\sigma} c_{\boldsymbol{\pi}} \mathfrak{a}\right)^{\prime \prime} \subseteq\left(c_{\sigma} \overline{\mathfrak{a}}\right)^{\prime}, \\
\left(c_{\sigma} \overline{\mathfrak{a}}\right)^{\prime \prime} \supseteq\left(c_{\sigma} c_{\boldsymbol{\pi}} \mathfrak{a}\right)^{\prime} \supseteq\left(c_{\sigma} \overline{\mathfrak{a}}\right)^{\prime \prime} \supseteq c_{\sigma} \overline{\mathfrak{a}} .
\end{gathered}
$$

If we match the last four statements in pairs, we get

$$
\begin{gathered}
\mathfrak{a}^{\prime \prime} \supseteq c_{\sigma} c_{\pi} \mathfrak{a}=\left(c_{\sigma} c_{\pi} \mathfrak{a}\right)^{\prime \prime}=\left(c_{\sigma} \overline{\mathfrak{a}}\right)^{\prime}, \\
\mathfrak{a}^{\prime} \subseteq c_{\sigma} \overline{\mathfrak{a}} \subseteq\left(c_{\sigma} \overline{\mathfrak{a}}\right)^{\prime \prime}=\left(c_{\sigma} c_{\pi} \mathfrak{a}\right)^{\prime} .
\end{gathered}
$$

But $\mathfrak{a} \subseteq c_{\sigma} c_{\pi} \mathfrak{a}$; hence $\mathfrak{a}^{\prime} \supseteq\left(c_{\sigma} c_{\pi} \mathfrak{a}\right)^{\prime}$ and $\mathfrak{a}^{\prime \prime} \subseteq\left(c_{\sigma} c_{\pi} \mathfrak{a}\right)^{\prime \prime}$, giving the theorem.

Corollary. A C-neutral ideal is normal if and only if $\mathfrak{a}=\mathfrak{a}^{\prime \prime}$.

The proof is obvious.

In a Boolean algebra all elements are neutral, so that $C C A=A$ for all $A \subseteq L$; if $\mathfrak{a}$ is a $\sigma$-ideal and $x, y \in C \mathfrak{a}$, then $\bar{x}+\bar{y} \in \mathfrak{a}$, and $x y \in C \mathfrak{a}$, and any ideal is $C$-neutral. Thus our definition of normality is a proper generalization of Stone's $\left(\mathfrak{a}^{\prime \prime}=\mathfrak{a}\right)$ in a Boolean algebra. Henceforth we may write $\mathfrak{a}^{\prime}$ for $c_{\sigma} \dot{\bar{a}}$, $\mathfrak{a}^{\prime \prime}$ for $c_{\sigma} c_{\pi} \mathfrak{a}, \overline{\mathfrak{a}}^{\prime}$ for $c_{\pi} \mathfrak{a}$, and $\overline{\mathfrak{a}}^{\prime \prime}$ for $c_{\pi} c_{\sigma} \overline{\mathfrak{a}}$.

Lemma 10. If $b_{1} \equiv b_{2}(\mathfrak{a})$ and $b_{1} \equiv b_{2}\left(\mathfrak{a}^{\prime}\right)$, then $b_{1}=b_{2}$. Similarly for $\mathfrak{a}^{\prime}$ and $\mathfrak{a}^{\prime \prime}$.

For take $a_{3}$ complementary to $b_{1} b_{2}$ in $b_{1}+b_{2}: a_{3} b_{1} b_{2}=O, a_{3}+b_{1} b_{2}=b_{1}+b_{2}$. Now $b_{1} b_{2} \equiv b_{i} \equiv b_{1}+b_{2}$ by either ideal, hence $a_{3}=a_{3}\left(b_{1}+b_{2}\right) \equiv a_{3} b_{1} b_{2}=O$ by either ideal, i.e., $a_{3} \in \mathfrak{a} \cap \mathfrak{a}^{\prime}$, so that $a_{3}=O$. Thus $b_{1} b_{2} \leqq b_{i} \leqq b_{1}+b_{2}=b_{1} b_{2}$, hence $b_{1}=b_{1} b_{2}=b_{2}$.

Lemma 11. If $a^{\prime} \in \mathfrak{a}^{\prime}$, then $x \equiv a^{\prime}$ if and only if $x=a+a^{\prime}$ with $a \in \mathfrak{a}$; $a^{\prime}+a_{1}=a^{\prime}+a_{2}$ if and only if $a_{1}=a_{2}$, so that $\left(a^{\prime}\right)^{*}$ is isomorphic with $a$ by $a^{\prime}+a \longleftrightarrow a$.

For there is a $t \in \overline{\mathfrak{a}}$ with $x t=a^{\prime} t=a^{\prime}$. Let $a$ be a complement of $x t$ in $x$. 
By Lemma $7 a \in \mathfrak{a}$ and hence $x=x t+a=a^{\prime}+a$. If $a^{\prime}+a_{1}=a^{\prime}+a_{2}$ with $a_{i} \in \mathfrak{a}$, then $a_{1}+a_{2} \in \mathfrak{a}$, and therefore

$$
\begin{aligned}
a_{1} & =a_{1}+a^{\prime}\left(a_{1}+a_{2}\right)=\left(a_{1}+a^{\prime}\right)\left(a_{1}+a_{2}\right)=\left(a_{2}+a^{\prime}\right)\left(a_{1}+a_{2}\right) \\
& =a_{2}+a^{\prime}\left(a_{1}+a_{2}\right)=a_{2} .
\end{aligned}
$$

Lemma 12. For any $a \in \mathfrak{a}, a^{\prime} \in \mathfrak{a}^{\prime}$ and $x \in L$ we have $x\left(a+a^{\prime}\right)=x a+x a^{\prime}$.

For $x\left(a+a^{\prime}\right) \equiv x a^{\prime} \in \mathfrak{a}^{\prime}$; hence Lemma 11 yields an $a_{1} \in \mathfrak{a}$ for which $x\left(a+a^{\prime}\right)=x a^{\prime}+a_{1}$. Thus $x a \equiv a_{1}\left(\mathfrak{a}^{\prime}\right)$. But $x a \equiv a_{1}(\mathfrak{a})$, since both are in $\mathfrak{a}$. Consequently, by Lemma $10, a_{1}=x a$.

We now define $A+B$ to mean the set of all $a+b$ with $a \in A, b \in B$. Similarly for $A B$.

Lemma 13. If $\mathfrak{b}$ is a $C$-neutral ideal $\supseteq \mathfrak{a}$, then $\mathfrak{a}+\mathfrak{b}^{\prime}$ is a $C$-neutral $\sigma$-ideal with $\mathfrak{a} \mathfrak{b}^{\prime}$ as its complement, and $\left(\mathfrak{a}+\mathfrak{b}^{\prime}\right)^{\prime}=\mathfrak{a}^{\prime} \cap \mathfrak{b}^{\prime \prime}$.

For $\mathfrak{b}^{\prime} \subseteq \mathfrak{a}^{\prime}$ and $\left(a_{1}+b_{1}^{\prime}\right)+\left(a_{2}+b_{2}^{\prime}\right)=\left(a_{1}+a_{2}\right)+\left(b_{1}^{\prime}+b_{2}^{\prime}\right)$; if $x \leqq a+b^{\prime}$, since $b^{\prime} \in \mathfrak{b}^{\prime} \subseteq \mathfrak{a}^{\prime}$, Lemma 12 gives $x=x\left(a+b^{\prime}\right)=x a+x b^{\prime}$, with $x a \in \mathfrak{a}, x b^{\prime} \in \mathfrak{b}^{\prime}$. Thus $\mathfrak{a}+\mathfrak{b}^{\prime}$ is an ideal. Now let $x \in C a+b^{\prime}$. Since $a+b^{\prime} \equiv b^{\prime} \in \mathfrak{b}^{\prime} \subseteq \mathfrak{a}^{\prime}$, and since, by Lemma 4 , we may take $x \leqq t^{\prime} \in C b^{\prime}$, it follows that $t^{\prime} \in \overline{\mathfrak{b}}^{\prime}$ and $x \equiv t^{\prime}$. Thus there is an $a_{1} \in \mathfrak{a}$ for which $x+a_{1}=t^{\prime}+a_{1}=t^{\prime}$, since $\overline{\mathfrak{b}}^{\prime} \subseteq \overline{\mathfrak{a}}^{\prime}$. But $x+a_{1}$ over $x$ has a complement $t \in \overline{\mathfrak{a}}$ by Lemma 7 ; hence $x=t t^{\prime} \in \overline{\mathfrak{a}} \overline{\mathfrak{b}}^{\prime}$. It follows that $C\left(\overline{\mathfrak{a}} \overline{\mathfrak{b}}^{\prime}\right) \subseteq \mathfrak{a}+\mathfrak{b}^{\prime}$, and, dually that $C\left(\mathfrak{a}+\mathfrak{b}^{\prime}\right) \subseteq \overline{\mathfrak{a}}^{\prime} \overline{\mathfrak{b}}^{\prime}$, so that $C C\left(\mathfrak{a}+\mathfrak{b}^{\prime}\right) \subseteq \mathfrak{a}+\mathfrak{b}^{\prime}$, and dually for $\overline{\mathfrak{a}} \overline{\mathfrak{b}}^{\prime}$; thus $\overline{\mathfrak{a}} \overline{\mathfrak{b}}^{\prime}=C C \overline{\mathfrak{a}}^{\prime} \overline{\mathfrak{b}}^{\prime} \subseteq C\left(\mathfrak{a}+\mathfrak{b}^{\prime}\right) \subseteq \overline{\mathfrak{a}} \overline{\mathfrak{b}}^{\prime}$, and the first statement follows.

If $x \in \mathfrak{a}^{\prime} \cap \mathfrak{b}^{\prime \prime}$, then $x a=O$ and $x b^{\prime}=O$ for all $a, b^{\prime}$; by Lemma 12, $x\left(a+b^{\prime}\right)=O$, and $x \in\left(\mathfrak{a}+\mathfrak{b}^{\prime}\right)^{\prime}$.

Finally, if $x\left(a+b^{\prime}\right)=O$, then $x a=x b^{\prime}=O$, and $x \in \mathfrak{a}^{\prime} \cap \mathfrak{b}^{\prime \prime}$, q. e. d.

Corollary. If $\mathfrak{b}$ is a C-neutral ideal $\supseteq \mathfrak{a}$, then $\left(\mathfrak{b}^{\prime \prime}\right)^{*}=\left(\mathfrak{a}+\mathfrak{b}^{\prime}\right)^{* \prime}$.

For if $x^{*} \in\left(\mathfrak{b}^{\prime \prime}\right)^{*}$, then $x \in \mathfrak{b}^{\prime \prime}$ by Theorem 4 , since $\mathfrak{b}^{\prime \prime} \supseteq \mathfrak{a}^{\prime \prime} \supseteq \mathfrak{a}$. Therefore $x b^{\prime}=O$ for all $b^{\prime} \in \mathfrak{b}^{\prime}$. Hence $x\left(a+b^{\prime}\right) \equiv O$ for all $a \in \mathfrak{a}$ and $b^{\prime} \in \mathfrak{b}^{\prime}$. Consequently,

$$
x^{*}\left(a+b^{\prime}\right)^{*}=\left(x\left(a+b^{\prime}\right)\right)^{*}=O^{*}
$$

and hence $x^{*} \in\left(\mathfrak{a}+\mathfrak{b}^{\prime}\right)^{* \prime}$.

If $x^{*} \in\left(\mathfrak{a}+\mathfrak{b}^{\prime}\right)^{* \prime}$, then as before, $x\left(a+b^{\prime}\right) \equiv O$, and $x b^{\prime} \equiv O$ for all $b^{\prime} \in \mathfrak{b}^{\prime} ;$ but $x b^{\prime} \in \mathfrak{b}^{\prime} \subseteq \mathfrak{a}^{\prime}$ and Lemma 10 gives $x b^{\prime}=O$. Hence $x \in \mathfrak{b}^{\prime \prime}$, and $x^{*} \in\left(\mathfrak{b}^{\prime \prime}\right)^{*}$.

Lemma 14. If $\mathfrak{a}$ is normal and $b x \in \mathfrak{a}$ for all $b \in \mathfrak{b}$, then $b^{\prime \prime} x \in \mathfrak{a}$ for all $b^{\prime \prime} \in \mathfrak{b}^{\prime \prime}$.

For $a^{\prime} b x=O$ for all $a^{\prime} \in \mathfrak{a}^{\prime}$ and $b \in \mathfrak{b}$; hence $a^{\prime} x \in \mathfrak{b}^{\prime}$ for all $a^{\prime} \in \mathfrak{a}^{\prime}$; therefore $b^{\prime \prime} a^{\prime} x=O$ for all $b^{\prime \prime} \in \mathfrak{b}^{\prime \prime}$ and $a^{\prime} \in \mathfrak{a}^{\prime}$, so that $b^{\prime \prime} x \in \mathfrak{a}^{\prime \prime}=\mathfrak{a}$ for all $b^{\prime \prime} \in \mathfrak{b}^{\prime \prime}$.

COROLlary. If $\mathfrak{a}$ is normal and $\mathfrak{b}$ is a C-neutral ideal $\supseteq \mathfrak{a}$, then $\mathfrak{b}^{* \prime}=\mathfrak{b}^{\prime \prime * \prime}$.

The proof is obvious. 
Theorem 7. If $\mathfrak{a}$ is a $C$-neutral ideal and $\mathfrak{b}$ is a $C$-neutral ideal $\supseteq \mathfrak{a}$, then:

1. If $\mathfrak{b}$ is normal, so is $\mathfrak{b}^{*}$.

2. If $\mathfrak{a}$ is normal and $\mathfrak{b}^{*}$ is normal, so is $\mathfrak{b}$.

3. If $a$ is normal, the correspondence of Theorem 4 preserves normality in both directions.

For 1 . By the corollary to Lemma 13 , if $\mathfrak{b}$ is normal, then $\mathfrak{b}^{*}=\left(\mathfrak{b}^{\prime \prime}\right)^{*}$ $=\left(\mathfrak{a}+\mathfrak{b}^{\prime}\right)^{* \prime}$, so that $\mathfrak{b}^{*}$ is normal.

2. By the last corollary, if $\mathfrak{a}$ is normal and $\mathfrak{b}^{*}$ is normal, then, using the first part of the proof, $\left(\mathfrak{b}^{\prime \prime}\right)^{*}=\left(\mathfrak{b}^{\prime \prime}\right)^{* \prime \prime}=\left(\mathfrak{b}^{*}\right)^{\prime \prime}=\mathfrak{b}^{*}$. Hence, by Theorem 4, $\mathfrak{b}^{\prime \prime}=\mathfrak{b}$.

3. The proof is now obvious.

We note that it is not hard to show, using Lemma 3 in $L(a, I)$ and Lemma 9, that $a^{*}$ can have a g.l.b. $a$ and an l.u.b. if and only if $a$ is principal; and that $L / \mathfrak{a}$ is isomorphic with $\mathfrak{a}^{\prime}$ if and only if $\mathfrak{a}^{\prime}$, and hence $\mathfrak{a}$, is principal. It also follows (using $\mathfrak{b}=\mathfrak{a}$ in Lemma 13 ) that

$$
\left(\mathfrak{a}+\mathfrak{a}^{\prime}\right)^{\prime}=\{O\}, \quad\left(\overline{\mathfrak{a}} \overline{\mathfrak{a}}^{\prime}\right)^{\prime}=\{I\}, \quad\left(\mathfrak{a}+\mathfrak{a}^{\prime}\right)^{\prime \prime}=L, \quad\left(\overline{\mathfrak{a}} \overline{\mathfrak{a}}^{\prime}\right)^{\prime \prime}=L,
$$

so that $a+\mathfrak{a}^{\prime}$ is normal if and only if $a+\mathfrak{a}^{\prime}=L$, which is possible if and only if $\mathfrak{a}$ is principal (for $\mathfrak{a}^{\prime} \cap \mathfrak{a}^{\prime \prime}=\{O\}$ ). Thus $\mathfrak{a}+\mathfrak{a}^{\prime}$ is not normal if $\mathfrak{a}$ is not principal, even if $\mathfrak{a}$ is normal. In Stone's terminology, $\mathfrak{a}+\mathfrak{a}^{\prime}$ is a barrier ideal in such a case. Stone has pointed out that the homomorphism $L \rightarrow L^{*}$ need not preserve normal ideals (we have needed $\mathfrak{b} \supseteq \mathfrak{a}$ to obtain such a correspondence). Indeed, using $\mathfrak{b}=\mathfrak{a}$ in the corollary to Lemma 13 we see that, if $\mathfrak{a}$ is normal, $\left(\mathfrak{a}+\mathfrak{a}^{\prime}\right)^{* \prime}=\left(\mathfrak{a}^{\prime \prime}\right)^{*}=\mathfrak{a}^{*}=O^{*}$, so that $\left(\mathfrak{a}^{\prime}\right)^{*}$ is not normal if $\mathfrak{a}$ is not principal (we have already seen that $\mathfrak{a}+\mathfrak{a}^{\prime}$ is not normal, so that we are not contradicting Theorem 7$)$, for $\left(\mathfrak{a}+\mathfrak{a}^{\prime}\right)^{*}=\left(\mathfrak{a}^{\prime}\right)^{*}$, and hence $\left(\mathfrak{a}^{\prime}\right)^{* \prime \prime}=L^{*}$; but $\left(\mathfrak{a}^{\prime}\right)^{*}=L$ only if $\mathfrak{a}+\mathfrak{a}^{\prime}=L$.

$L$ is called ideally irreducible if it contains no normal $C$-neutral $\sigma$-ideal $(\pi$-ideal $) \neq O^{\sigma}$ or $I^{\sigma}\left(O^{\pi}\right.$ or $\left.I^{\pi}\right)$. Every ideally irreducible $L$ is also irreducible. If $L$ is complete, the two concepts coincide.

Theorem 8. If $\mathfrak{a}$ is a normal $C$-neutral $\sigma$-ideal $(\pi$-ideal $) \neq O^{\sigma}, I^{\sigma}\left(O^{\pi}, I^{\pi}\right)$, then $L / \mathfrak{a}$ is ideally irreducible if and only if $\mathfrak{a}$ is maximal (prime) in the partially ordered set of normal $C$-neutral $\sigma$-ideals ( $\pi$-ideals).

This is an obvious consequence of Theorem 7.

By a quasi-norm on $L, r(x)$, we mean a positive (or monotone) modular functional. If it is sharply positive, we call it a norm. It is called normalized if $r(O)=0, r(I)=1$.

THEOREM 9. If $r(x)$ is a normalized quasi-norm, then the set of $x \in L$ for which $r(x)=1$ is a $C$-neutral $\pi$-ideal $\overline{\mathfrak{a}}$, and $\mathfrak{a}$ is the set of all $x \in L$ for which $r(x)=0$; furthermore, $a \equiv b(\mathfrak{a})$ if and only if $r(a b)=r(a+b)$. (This is an extension of Lemma 1 in $\S 87$, Birkhoff.) 
The proof falls into seven sections.

1. If $x \geqq y$ and $r(y)=1$, then $r(x) \geqq r(y)=1$, so that $r(x)=1$. If $r(x)=r(y)$ $=1$, then $r(x+y)=1$, so that

$$
r(x y)=r(x)+r(y)-r(x+y)=1 .
$$

Thus $\overline{\mathfrak{a}}$ is a $\pi$-ideal.

2. If $y \in C x$, then

$$
1=r(I)=r(x+y)=r(x)+r(y)-r(x y)=r(x)+r(y) .
$$

3. If $y, y^{\prime} \in C x$, then $r(y)=1-r(x)=r\left(y^{\prime}\right)$.

4. If $x \in \overline{\mathfrak{a}}$ and $x^{\prime} \in C C x$, then, $r\left(x^{\prime}\right)=r(x)=1 ; C C \mathfrak{a} \subseteq \mathfrak{a}$.

5. If $x \in C y$, then $x \in \bar{a}$ if and only if $r(y)=0$, by 2. Since, by the dual of 1 , the latter class is a $\sigma$-ideal, $C \bar{a}$ is a $\sigma$-ideal.

6. By 4 and $5, \overline{\mathfrak{a}}$ is $C$-neutral, and $\mathfrak{a}$ is the set of $x$ with $r(x)=0$.

7. If $a \equiv b(\mathfrak{a})$, then $a+b=a b+u$ where $u \in \mathfrak{a}$. But $r(u)=r(a b u)=0$. Therefore $r(a+b)=r(a b+u)=r(a b)+r(u)-r(a b u)=r(a b)$. If $r(a b)=r(a+b)$, take $u$ a complement of $a b$ in $a+b: a+b=a b+u, a b u=O$; then

$$
r(a+b)=r(a b)+r(u)-r(a b u)=r(a b)+r(u)
$$

so that $r(u)=0, u \in \mathfrak{a}$, and $a+b \equiv a b(\mathfrak{a})$.

CoROllaRY. If $a \equiv b(a)$, then $r(a)=r(b)$. If $a<b$ and $r(a)=r(b)$, then $a \equiv b(\mathfrak{a})$.

To prove the first part, suppose $a \equiv b(\mathfrak{a})$. Then

$$
a b \leqq\left\{\begin{array}{l}
a \\
b
\end{array}\right\} \leqq a+b
$$

yields

$$
r(a b) \leqq\left\{\begin{array}{l}
r(a) \\
r(b)
\end{array}\right\} \leqq r(a+b)=r(a b) ;
$$

hence $r(a)=r(a b)=r(b)$.

As for the second, let $u$ be a complement of $a$ in $b: b=a+u, a u=O$; then $r(a)=r(b)=r(a)+r(u), r(u)=0, u \in \mathfrak{a}$, and $a \equiv b(\mathfrak{a})$.

Theorem 10. If $r(x)$ is a quasi-norm in $L$, then $r\left(x^{*}\right)=r(x)$ is a norm in $L^{*}=L / \mathfrak{a}$, and every norm in $L^{*}$ determines a quasi-norm in $L$ by $r(x)=r\left(x^{*}\right)$. There is a $(1,1)$ correspondence between the norms of $L^{*}$ and the quasi-norms of $L$ which determine a.

The result is obvious except for the fact that $r\left(x^{*}\right)$ is sharply positive: if $x^{*}<y$, then $x^{*} y^{*}=x^{*} \neq y^{*}$, so that $x y \equiv x$ and $x \neq \equiv y$; there is then a $t \in \mathfrak{a}$ for which $x y t=x t, x t<y$, and hence $x t \neq \equiv y$. By the above corollary we must then have $r(x)=r(x t)<r(y)$, and consequently $r\left(x^{*}\right)<r\left(y^{*}\right)$. 
LEMMA 15. If $a \neq O$ or I is a central (i.e., complemented and neutral) element in $L$ (which need not be complemented) and $r(x)$ is a normalized norm in $L$, then there is another normalized norm in $L$ which differs from $r(x)$ either on $a$ or on $\bar{a}$.

For choose the numbers $m$ and $\bar{m}$ so that $m+\bar{m}=1, m>0, \bar{m}>0$, and $m \neq \bar{m}$. Each $x=a x+\bar{a} x$. Define

Then $\bar{R}(O)=O$ and

$$
\bar{R}(x)=m r(a x)+\bar{m} r(\bar{a} x) .
$$

$$
\bar{R}(I)=m r(a)+\bar{m} r(\bar{a})<r(a)+r(\bar{a})=1 .
$$

If $x<y$, then $a x \leqq a y$ and $\bar{a} x \leqq \bar{a} y$, and at least one of the equality signs does not hold. Thus

$$
\bar{R}(x)=m r(a x)+\bar{m} r(\bar{a} x)<m r(a y)+\bar{m} r(\bar{a} y)=\bar{R}(y) .
$$

Also

$$
\begin{aligned}
\bar{R}(x)+\bar{R}(y) & =m r(a x)+\bar{m} r(\bar{a} x)+m r(a y)+\bar{m} r(\bar{a} y) \\
& =m[r(a x+a y)+r(a x y)]+\bar{m}[r(\bar{a} x+\bar{a} y)+r(\bar{a} x y)] \\
& =m[r(a(x+y))+r(a x y)]+\bar{m}[r(\bar{a}(x+y))+r(\bar{a} x y)] \\
& =\bar{R}(x+y)+\bar{R}(x y) .
\end{aligned}
$$

Hence $\bar{r}(x)=\bar{R}(x) / \bar{R}(I)$ is a normalized norm over $L$. Now $\bar{R}(a)=m r(a)$ and $\bar{R}(\bar{a})=\bar{m} r(\bar{a})$; therefore $r(a)=\bar{r}(a)$ if and only if $m=\bar{R}(I)$, and $r(\bar{a})=\bar{r}(\bar{a})$ if and only if $\bar{m}=\bar{R}(I)$. Since $m \neq \bar{m}$, both cannot be true at the same time.

COROLlary. If the normalized quasi-norm $r(x)$ in the Boolean algebra $L$ determines the $\sigma$-ideal $\mathfrak{a}$, then $r(x)$ is uniquely determined by $\mathfrak{a}$ if and only if $\mathfrak{a}$ is prime.

For $L / \mathfrak{a}$ is a Boolean algebra, so that if it contains other elements beside $O$ and $I$, they will be central elements; in such a case $r\left(x^{*}\right)$ and hence $r(x)$ would not be unique. Hence, if $r(x)$ is unique, $L / \mathfrak{a}=\left\{O^{*}, I^{*}\right\}$, and a must be prime, since $L=\mathfrak{a} \cup \mathfrak{a}^{\prime}$. Conversely, if $\mathfrak{a}$ is prime, $r\left(x^{*}\right)$ must be unique (note that $r\left(O^{*}\right)=0$ and $r\left(I^{*}\right)=1$ ) and $r(x)$ is uniquely determined by $a$.

Wilcox and Smiley $\left({ }^{2}\right)$ have given continuity conditions on a norm of $L$ sufficient to assure isomorphism between the complete envelope $\bar{L}$ and the metrically complete envelope of $L$. Their conditions imply that if $a_{\alpha} \rightarrow A$ and $b_{\alpha} \rightarrow B$, then $r\left(a_{\alpha}\right) \rightarrow r(A), a_{\alpha}+b_{\alpha} \rightarrow A+B$, and so on. With these conditions, MacNeille's problem( $\left.{ }^{3}\right)$ is answered in the positive: $\bar{L}$ is modular, since it possesses a norm. Because of the existence of $O$ and $I$ in our lattices, the W.S. conditions reduce to the following definition.

(2) Annals of Mathematics, (2), vol. 40 (1939), p. 309.

(3) See Birkhoff's Lattice Theory, American Mathematical Society Colloquium Publications, vol. 25, 1940, p. 146 (3). 
A norm or quasi-norm in a lattice is called W.S. if for every $O \subset A \subseteq L$ we have

$$
\text { l.u.b. } r(a) \text { for } a \in A=\text { g.l.b. } r(b) \text { for } b \in c_{\pi} A \text {, }
$$

and

$$
\text { g.l.b. } r(a) \text { for } a \in A=1 \text {.u.b. } r(b) \text { for } b \in c_{\sigma} A \text {. }
$$

THEOREM 11. If the complemented modular $L$ has a W.S. norm, then $L$ is ideally irreducible if and only if its complete envelope, $\bar{L}$, is irreducible.

For suppose that $\bar{L}$ is reducible with central element $A$ and complement $\bar{A} \neq O, I$. Then $L \cap c_{\sigma} A$ and $L \cap c_{\pi} \bar{A}$ are normal. Since $L$ is dense in $\bar{L}$;

$$
A=\sum\left(L \cap c_{\sigma} A\right)=\prod\left(L \cap c_{\boldsymbol{\pi}} A\right),
$$

so that

$$
L \cap c_{\sigma} A \neq O^{\sigma}, I^{\sigma} .
$$

By Lemma 6 (which does not require complementation), $L \cap c_{\sigma} A$ and $L \cap c_{\pi} \bar{A}$ are complementary $C$-neutral ideals. Hence $L$ is ideally reducible.

Conversely, if $L$ is ideally reducible with $a \neq O^{\sigma}, I^{\sigma}$ a normal $C$-neutral $\sigma$-ideal, then $\mathfrak{a}^{\prime}=c_{\sigma} \overline{\mathfrak{a}}$ and $\overline{\mathfrak{a}}^{\prime}=c_{\pi} \mathfrak{a}$ by Theorem 6 and its dual. Hence $\sum \mathfrak{a}^{\prime}=\sum c_{\sigma} \overline{\mathfrak{a}}=\prod \overline{\mathfrak{a}}$ and $\prod_{\bar{a}^{\prime}}=\prod c_{\pi} \mathfrak{a}=\sum \mathfrak{a}$ by the following continuity argument and its dual: every $a^{\prime} \leqq$ every $\bar{a}$, therefore $\sum \mathfrak{a}^{\prime}=\sum c_{\sigma} \overline{\mathfrak{a}} \leqq \prod \overline{\mathfrak{a}}$; but

$$
r\left(\sum \mathfrak{a}^{\prime}\right)=\text { l.u.b. } r\left(a^{\prime}\right)=\text { g.l.b. } r(\bar{a})=r\left(\prod \bar{a}\right) ;
$$

hence $\sum \mathfrak{a}^{\prime}=\prod \overline{\mathfrak{a}}=\bar{A}$, say, and $\sum \mathfrak{a}=\prod \overline{\mathfrak{a}}^{\prime}=A$. Now if $a_{\alpha}^{\prime} \uparrow A$ and $a_{\alpha} \uparrow A$, where $a_{\alpha}^{\prime} \in \mathfrak{a}^{\prime}$ and $a_{\alpha} \in \mathfrak{a}$, then $O=a_{\alpha} a_{\alpha}^{\prime} \rightarrow A \bar{A}$. Consequently $A \bar{A}=O$ and dually $A+\bar{A}=I$.

By Lemma 12, $x\left(a_{\alpha}+a_{\alpha}^{\prime}\right)=x a_{\alpha}+x a_{\alpha}^{\prime}$; hence $x(A+\bar{A})=x A+x \bar{A}$ for every $x \in L$; therefore, finally, $X(A+\bar{A})=X A+X \bar{A}$ for every $X \in \bar{L}$. Thus

$$
X+Y=X A+X \bar{A}+Y A+Y \bar{A},
$$

and, since $\bar{L}$ is modular,

$$
A(X+Y)=A X+A Y
$$

and

$$
\bar{A}(X+Y)=\bar{A} X+\bar{A} Y .
$$

Consequently $A$ and $\bar{A}$ are complementary central elements. $\bar{L}$ is therefore reducible, q.e.d.

THEOREM 12. If $L$ is a complemented modular lattice with a W.S. norm, $r(x)$, and $\mathfrak{a}$ is a normal $C$-neutral $\sigma$-ideal $\neq O^{\sigma}$ or $I^{\sigma}$, then there is another norm, $s(x)$, differing from $r(x)$ either on $\mathfrak{a}$ or on $\mathfrak{a}^{\prime}$. 
For by Theorem $11, \sum \mathfrak{a}$ is central in $\bar{L}$. Hence Lemma 15 applies in $\bar{L}$, and $s(x)$ specializes to another norm for $L$ (which is a sublattice of $\bar{L}$ ).

COROLlaRY. If $L$ is a complemented modular lattice with a W.S. norm, $r(x)$, then $r(x)$ is uniquely determined (except for linear transformation) if and only if $L$ is ideally irreducible.

For $L$ fulfills von Neumann's continuity conditions (see Wilcox and Smiley). Hence, if $L$ is irreducible, it is a continuous geometry, and von Neumann's theorem tells us that $r(x)$ is unique. The converse follows from Theorems 11 and 12.

THEOREM 13. If the complemented modular $L$ has the W.S. quasi-norm, $r(x)$ whose determined ideal is $\mathfrak{a}$, then $\mathfrak{a}$ is normal.

For $\overline{\mathfrak{a}}^{\prime}=c_{\boldsymbol{\pi}} \mathfrak{a}$. Therefore

$$
c_{\sigma} \overline{\mathfrak{a}}^{\prime}=c_{\sigma} c_{\pi} \mathfrak{a}=\mathfrak{a}^{\prime \prime},
$$

and hence two applications of the continuity conditions yield

$$
\text { l.u.b. } r\left(a^{\prime \prime}\right)=\text { g.l.b. } r\left(\bar{a}^{\prime}\right)=\text { l.u.b. } r(a)=0 \text {. }
$$

Thus $r\left(a^{\prime \prime}\right)=0$, and $\mathfrak{a}^{\prime \prime}=\mathfrak{a}$.

THEOREM 14. If $L$ is a complemented modular lattice having $r(x)$ as a W.S. quasi-norm, and if the determined $\sigma$-ideal is $\mathfrak{a}$, then a uniquely determines $r(x)$ if and only if it is maximal (prime for the $\pi$-ideal) among the normal $C$-neutral ideals.

By the preceding results we have only to show that $r\left(a^{*}\right)$ is a W.S. norm in $L / \mathfrak{a}$. If $A^{*} \subseteq L / \mathfrak{a}$ and

$$
x \in c_{\pi} \cup a_{a^{*} \in A^{*}} a^{*}
$$

then $x \geqq$ every $a \in a^{*} \in A^{*}$, so that $x \in x^{*} \subseteq \cup c_{x} A^{*}$. Thus $c_{x} \cup A^{*} \subseteq \cup c_{\pi} A^{*}$. Similarly, $c_{\sigma} \cup A^{*} \subseteq \cup c_{\sigma} A^{*}$. Consequently,

$$
\text { l.u.b. } \begin{aligned}
r\left(a^{*}\right) \text { for } a^{*} \in A^{*} & =\text { l.u.b. } r(a) \text { for } a \in \cup A^{*} \\
& =\text { g.l.b. } r(b) \text { for } b \in c_{\pi} \cup A^{*} \\
& \geqq \text { g.l.b. } r(b) \text { for } b \in \cup c_{\pi} A^{*} \\
& =\text { g.l.b. } r(b) \text { for } b^{*} \in c_{\pi} A^{*} .
\end{aligned}
$$

The opposite inequality is obvious. Since the dual follows similarly, we have our result.

Columbia University, NEW YoRK, N.Y. 